\title{
Quelques études océanographiques présentées à l'Assemblée d'Helsinki
}

\author{
Oceanographical studies \\ presented at the Helsinki Congress
}

\author{
PAR B. SAINT-GUILY
}

MAITRE DE RECHERGHES AU C.N.R.S.

\begin{abstract}
Parmi les très nombreuses communications, quatre seuiement sont retenues et exposées très brièvement. Puis, à propos des travanx du Dr J.A. Knauss sur les courants équatoriaux du Pacifique, l'explication dynamique du fameux courant de Cromwell est abordée.
\end{abstract}

\begin{abstract}
Only four out of the very many papers received were selected and presented as very brief exposés. The dynamic explanation of the wellknown Cromwell current was then undertaken in connection with Dr. J.A. Knauss's work on equatorial currents in the Pacific Ocean.
\end{abstract}

La $12^{\text {e }}$ Assemblée générale de l'U.G.G.I. qui s'est tenue à Helsinki du 26 juillet au 6 août derniers, a réuni treize cents participants appartenant aux trente-sept pays de l'Union. Les locaux de l'Université n'ont pas suffi pour les travaux des sept associations et le Comité d'organisation a dû faire les plus grands efforts, en particulier pour loger les participants. $\mathrm{Si}$, à l'avenir, on veut renouveler des réunions de cette importance, il ne sera plus possible de le faire, si ce n'est dans quelques grandes capitales.

Il n'est peut-être pas inutile de dire que l'Association d'Océanographie Physique a changé en partie son bureau. Le nouveau président est le Dr. Deacon (Anglais); les vice-présidents sont, l'un Russe, l'autre Finlandais, et un Français, l'Ingénieur en chef $M$. Eyries, est secrétaireadjoint.

Les séances de travail ont distribué un nombre considérable de communications qui ont été groupées sous les rubriques suivantes :

- Déversement de l'eau froide arctique sur les seuils sous-marins de l'Atlantique Nord.

- Courants.

(*) Présentées à la $12^{\mathrm{e}}$ Assemblée générale de l'U.G.G.I. à Helsinki.
-. Marées dans les estuaires.

- Niveau moyen de la mer.

- Ondes de tempête et tsunamis.

- Energie reçue par la mer sous forme de rayonnement.

- Circulation du $\mathrm{CO}_{2}$ dans l'atmosphère et l'océan, etc.

Il ne peut être question de passer en revue les sujets des communications présentées, ni même les principaux dans chaque rubrique. Il faut nécessairement faire un choix plus ou moins arbitraire et limité. Je vais donc présenter très brièvement les sujets de quatre communications. La dernière me permettra d'ajouter quelques mots sur le nouveau et fameux courant équatorial sub-superficiel de Cromwell.

\section{$*^{*} *$}

Une communication de deux Américains, W. Munk et R. Haubrich, était intitulée : «Spectre des oscillations du niveau de la mer entre 0,05 et 1 cycle par an »: Les auteurs ont exposé les résultats d'une analyse spectrale du niveau moyen mensuel, faite à partir d'observations de la marée d'une durée de plus d'un siècle. Cette 


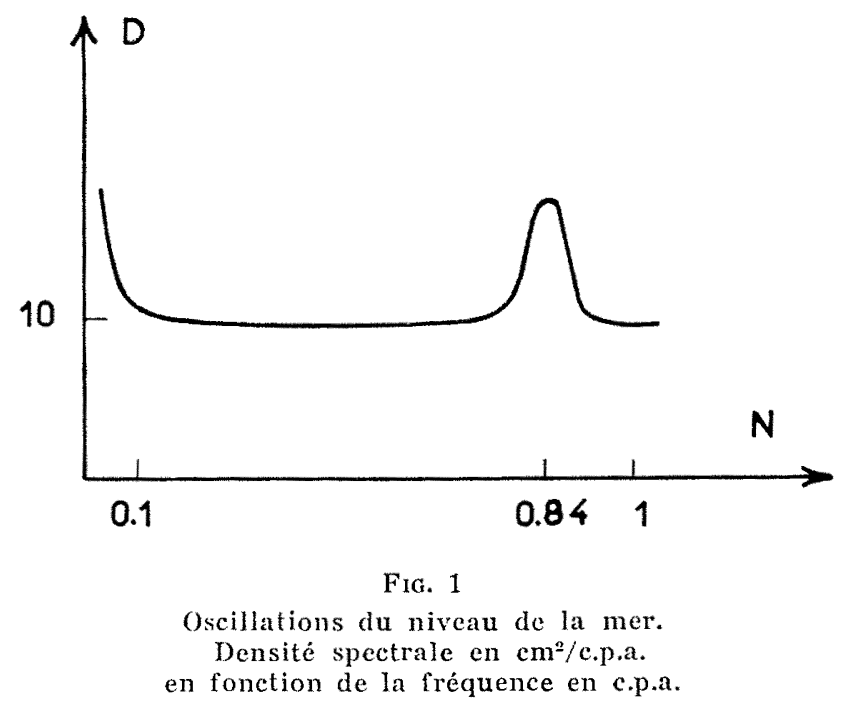

analyse spectrale a donné une courbe représentant la densité spectrale en fonction de la fréquence (fig. 1).

La densité spectrale est exprimée en $\mathrm{cm}^{2}$ par cycles par an, la fréquence, en cycles par an. On constate sur cette courbe une valeur uniforme de la densité spectrale entre 0,1 et 1 cycle par an, avec seulement un maximum pour 0,84 cycle par an. Cette fréquence correspond à une période de 14 mois, que l'on sait être celle de la libre nutation de la terre. En effet, si un solide est en rotation autour de son axe principal d'inertie de plus grand moment, son mouvement est stable. Une légère perturbation de ce mouvement fait décrire à l'axe de rotation un cône autour de l'axe d'inertie de plus grand moment; et la période de cette nutation est pour la terre, supposée rigide, de 305 jours. Mais l'élasticité du globe intervient, et cette période se trouve allongée jusqu'à 14 mois. Ainsi cctte nutation produit une oscillation du niveau de la mer. Au-dessous de 0,1 cycle par an, la courbe croît rapidement, probablement en raison des mouvements verticaux des continents. Le Dr. Munk et les physiciens de La Jolla (Californie) ont étudié systématiquement le spectre des oscillations du niveau de la mer, et ont obtenu ainsi des résultats fort intéressants.

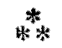

Une autre communication, de C.L. Pekeris et M. Dishon (Israël), nous a présenté des « marées théoriques obtenues à l'aide d'une calculatrice électronique de l'Institut Weizmann ». Il s'agit de calculs effectués à partir des équations de Laplace. Ceux-ci ont d'abord porté sur des mo- dèles d'océans de forme simplifiée et de profondeur constante. Les auteurs ont obtenu des résultats en accord avec les solutions données par Doodson, Goldsbrough et Rossiter. Les marées calculées pour la mer Rouge et pour la mer Noire concordent avec les observations et les solutions approchées de Proudman et Grace. Enfin, les auteurs ont entrepris le calcul des principaux constituants harmoniques de l'océan mondial (c'est-à-dire de l'ensemble des océans).

\section{$* *$}

Sur les « ondes internes », nous avons eu deux intéressantes communications : l'une de C. Cox, l'autre de E.C. La Fond. Je ne parlerai pas de la première qui était consacrée surtout à la description d'un nouveau dispositif d'enregistrement des fluctuations de température. La seconde portait sur les ondes internes (thermiques) près de la surface. Une chaîne de thermistors, mise au point au Laboratoire de San Diego, permet d'enregistrer de façon continue la profondeur des isothermes voisines de la surface en fonction du temps. Des observations faites avec cet instrument au sud de la Californie ont mis en évidence des oscillations de la thermocline d'été d'une amplitude de 5 pieds et d'une période supérieure à 7,6 minutes. A ces mouvements de la thermocline est liée une circulation convergente au-dessus des creux de l'onde interne; on a donc en surface, à cet endroit et par beau temps, une concentration de petites matières organiques qui forment des bandes lisses parfaitement visibles. La vitesse et la direclion de propagation de ces ondes internes a été déterminée par l'enregistrement continu de la température en trois points. Les mesures effectuées montrent que les ondes internes se réfractent vers les faibles profondeurs (elles « sentent » donc le fond comme la houle), et qu'elles se propagent vers la côte à la vitesse d'environ $15 \mathrm{~cm} / \mathrm{s}$. Quand ces ondes se trouvent près du fond, les crêtes deviennent assez aiguës; et quelques observations de structures therniques inversées font penser qu'il se produit de temps a autre un déferlement.

J'en viens maintenant aux travaux du Dr. J.A. Knauss sur les « courants équatoriaux du Pacifique ». Sa communication portait sur le contre-courant équatorial qui, à la latitude de $7^{\circ}$ nord environ, porte à l'est. C'est un courant relativement étroit ( 300 à $500 \mathrm{~km}$ ), compris entre les larges courants équatoriaux nord et sud qui 


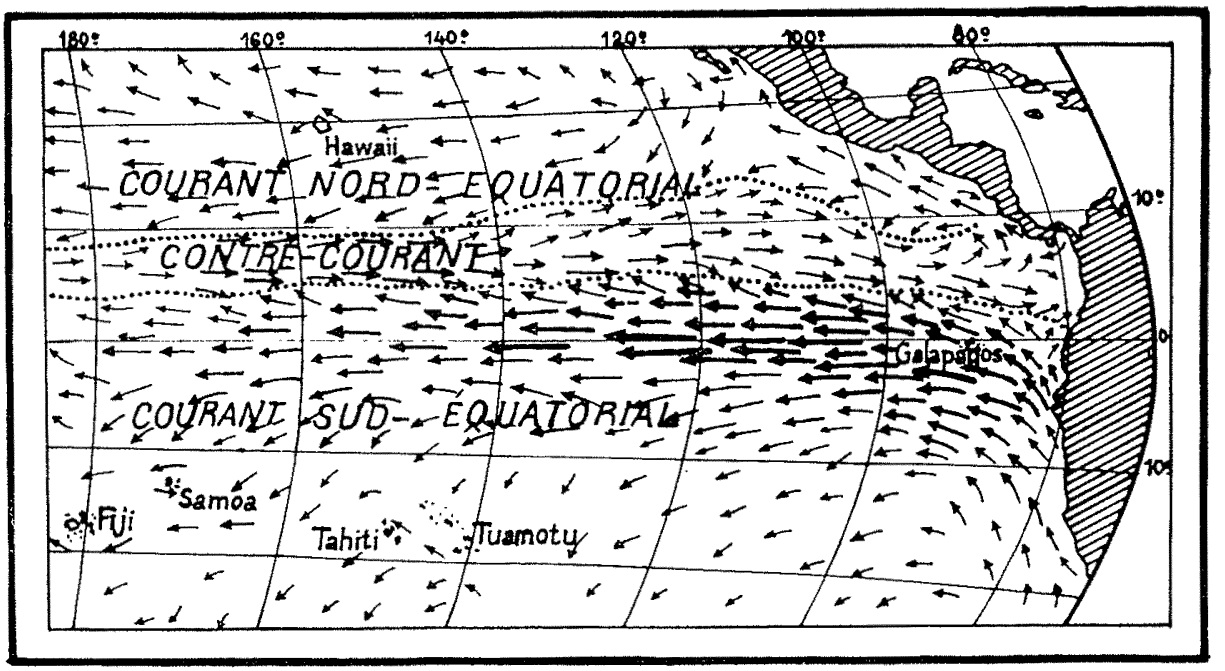

FIG. 2

Courants équatoriaux de l'océan Pacifique.

portent à l'ouest (fig. 2). Son existence est due à un minimum de la tension du vent en surface, minimum qui constitue une frontière entre les vents alizés du nord et du sud : la zone des calmes ou « pot-au-noir ». La vitesse du courant est maximale près de la surface et diminue à une profondeur de l'ordre de 100 mètres, au niveau de la thermocline. En surface, la vitesse est de 30 à $60 \mathrm{~cm} / \mathrm{s}$. Les observations montrent, de plus, que la composante turbulente de la vitesse augmente proportionnellement à la vitesse moyenne. Au-dessous de la thermocline, les choses sont moins claires : en effet, le débit, de 0 à 1500 mètres, varie de 60 millions de $\mathrm{m}^{3} / \mathrm{s}$ en 1958 à 1 million de $\mathrm{m}^{3} / \mathrm{s}$ en 1959 .

Le Dr. Knauss a étudié par ailleurs, de façon très détaillée et précise, le fameux courant découvert par Cromwell en 1954. Il s'agit d'une veine de courant de l'Océan Pacifique centrée sur l'équateur à une profondeur de l'ordre de 100 mètres, et portant à l'est, donc en sens inverse du courant de surface - courant sud-équatorial et du courant sous-jacent. Ce courant a une largeur de 200 à 300 kilomètres et une épaisseur d'environ 200 mètres. Les vitesses y atteignent $1,5 \mathrm{~m} / \mathrm{s}$ et le débit moyen est de l'ordre de 40 millions de $\mathrm{m}^{3} / \mathrm{s}$, soit un débit inférieur à celui du Gulf-Stream, mais du même ordre (fig. 3). J.A. Knauss a montré que ce courant était sensiblement géostrophique entre $2^{\circ}$ et $0,5^{\circ}$ de latitude nord et sud; mais ceci ne donne pas l'origine du courant. Théoriquement, on rencontre une difficulté due à l'impossibilité de linéariser les équations du mouvement à l'équateur. Le paramètre de Coriolis $\lambda=2 \omega$ sin to tend en effet vers zéro pour $\varphi=0$, si bien que les termes d'inertie (non linéaires) ne sont plus négligeables. Les travaux théoriques de J.G. Charney et G. Veronis ont montré que le vent d'est semble bien la cause de ce courant d'ouest; bien enlendu, ce n'est possible que dans un océan limité par des frontières méridiennes. Avec une

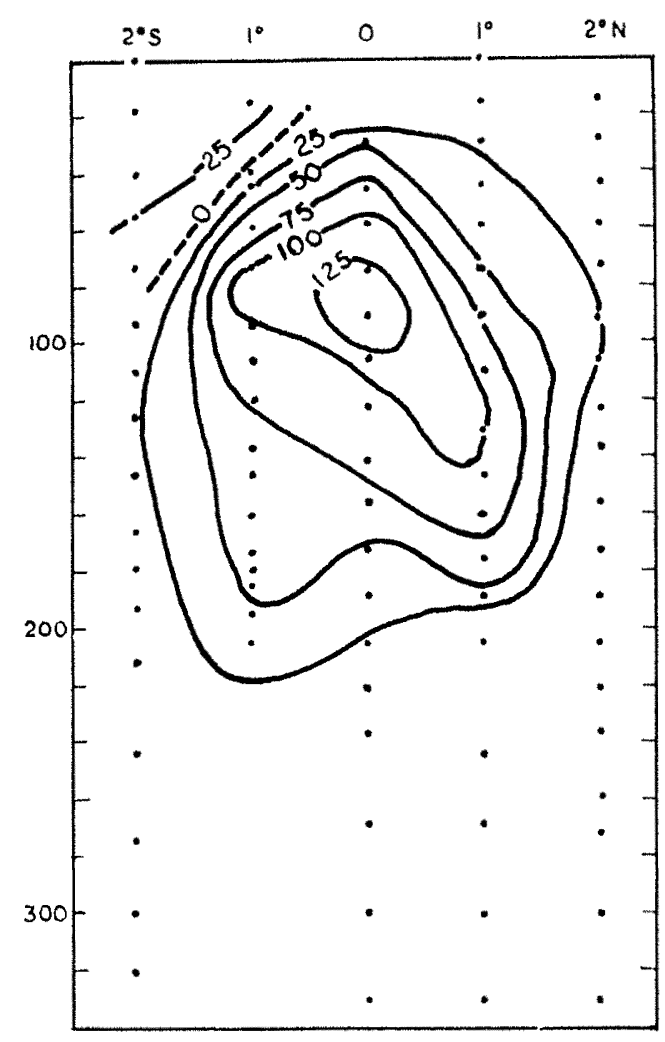

FiG. 3

Courant de Cromwell. Coupe verticale sur le méridien 140 ${ }^{\circ} W$ donnant les lignes d'égale vitesse; chaque point correspond à une observation, Les profondeurs sont exprimées en mètres, les vitesses en $\mathrm{cm} / \mathrm{s}$. 
tension du vent de $0,5 \mathrm{dyne} / \mathrm{cm}^{2}$, qui donne une pente de $3 \mathrm{~cm}$ pour $1000 \mathrm{~km}$, et pour un facteur de lurbulence de l'ordre de $15 \mathrm{~cm}^{2} / \mathrm{s}$, J.G. Charney a obtenu des vitesses comparables aux observations. Il écrit les équations suivantes :

$$
\begin{aligned}
\frac{\partial u}{\partial t}+v \frac{\partial u}{\partial !}+w \frac{\partial u}{\partial z}-\lambda v & =-\frac{1}{\rho} \frac{\partial p}{\partial x}+\frac{\mu}{\rho} \frac{\partial^{2} v}{\partial z^{2}}, \\
\frac{\partial v}{\partial t}+v \frac{\partial v}{\partial !}+w \frac{\partial v}{\partial z}+\lambda u & =-\frac{1}{\rho} \frac{\partial p}{\partial y}+\frac{\mu}{\rho} \frac{\partial^{2} v}{\partial z^{2}}, \\
\frac{\partial v}{\partial y}+\frac{\partial w}{\partial z} & =0,
\end{aligned}
$$

dans un système $\mathrm{O} x, \mathrm{O} y, \mathrm{O} z$ orienté est, nord, zénith; il suppose la pression égale à la pression hydrostatique, et $\lambda=(2 \omega / r) y$. Quelques transformations conduisent à un système un peu plus simple dont il a obtenu une solution asymptotique (pour un temps très grand), par un calcul aux différences finies effectué sur une calculatrice I.B.M. 704. De son côté, G. Veronis fait intervenir en plus la diffusion turbulente verticale et une densité variable; il part du système d'équations :

$$
\begin{aligned}
& u \frac{\partial u}{\partial x}+v \frac{\partial u}{\partial y}+w \frac{\partial u}{\partial z}-\lambda v=-\frac{1}{\rho} \frac{\partial p}{\partial x} \\
& +\frac{\mu}{\rho} \frac{\partial^{2} \mu}{\partial z^{2}} \\
& \lambda u=-\frac{1}{\varrho} \frac{\partial p}{\partial y}, \\
& 0=-\frac{1}{\rho} \frac{\partial p}{\partial z}+g x \mathrm{~T} \\
& \frac{\partial u}{\partial x}+\frac{\partial v}{\partial y}+\frac{\partial w}{\partial z}=0 \\
& u \frac{\partial \mathrm{T}}{\partial x}+v \frac{\partial \mathrm{T}}{\partial y}+w \frac{\partial \mathrm{T}}{\partial z}=\mu \frac{\partial^{2} \mathrm{~T}}{\partial z^{2}}
\end{aligned}
$$

Le système de coordonnées est le même; $\alpha$ est le coefficient d'expansion thermique et $T$ la température. Il utilise l'approximation de Boussinesq dans laquelle on retient les variations de densité seulement dans le terme gravitationnel et dans les termes de convection et de diffusion. Il effectue les développements suivants au voisinage de l'équateur :

$$
\begin{aligned}
(u, w, p, \mathrm{~T}) & =\sum_{n=0}^{\infty}\left(u_{2 n}, w_{2 n}, p_{2 n}, \mathrm{~T}_{2 n}\right) y^{2 n}, \\
v & =\sum_{n=0}^{\infty} v_{2 n+1} y^{2 n+1}
\end{aligned}
$$

et fait ensuite un calcul d'approximation assez étrange dans lequel il remplace une partie des termes de convection non linéaires par leur valeur moyenne sur toute l'épaisseur d'eau. Cette valeur moyenne est elle-même déterminée en moyennant les valeurs finales obtenues. Veronis a ainsi trouvé un courant d'une épaisseur de l'ordre de 120 mètres et une vitesse maximale de l'ordre de $1,70 \mathrm{~m} / \mathrm{s}$. Tous ces calculs sont peu satisfaisants, car ils comportent des approximations grossières. Ils fournissent seulement des ordres de grandeur et une image de la réalité déformée (du genre de celle que l'on avait au moyen âge à travers les petites vitres en cul de bouteille!); mais cette vision s'améliorera certainement dans l'avenir.

La stabilité de ce courant de Cromwell est due essentiellement à la variation du paramètre de Coriolis avec la latitude, variation qui est maximale à l'équateur. Un calcul portant sur une veine de courant infiniment mince le montre aisément. Mais ces questions nous font entrer dans le vif de la dynamique des courants marins et quitter Helsinki; j'en reste donc là.

\section{BIBLIOGRAPHIE}

Knauss J.A. - Measurements of the Cromwell current. Deep Sea Research, 6, no 4, 1960, p. 265-286.

Charney J.G. - Non-linear theory of a wind-driven homogeneous layer near the Equator. Deep Sea Research, 6, $\mathrm{n}^{\circ} 4,1960$, p. 303-317.

VERoNis G. - An approximate theorical analysis of the equatorial undercurrent. Deep Sea Research, 6, $\mathrm{n}^{\circ} 4,1960$, p. 318-327.
M. le Président remercie M. Salnt-Gurly qui a choisi, parmi tous les travaux présentés au Symposium d'Helsinki, trois ou quatre mémoires d'un particulier intérêt.

M. Serra demande comment le courant de Cromwell a été découvert.

A cette question, M. Lacombe répond qu'il pense que la découverte du courant de Cromwell a été faite au cours de mesures directes dans la région orientale du Pacifique équatorial qui est la zone d'élection des études de la Scripps Institution of Oceanography de la Jolla. Le bruit a couru à l'Assemblée d'Helsinki qu'un navire soviétique de recherches aurait découvert dans l'Atlantique un courant de même nature. La similitude de la disposition zonale des courants marins dans le Pacifique et l'Atlan- 
tique, malgré des formes différentes de leurs limites, plaide en faveur d'une telle existence. Rien de comparable n'a encore été dit concernant l'océan Indien.

M. DE Rouvilte demande quel est le méridien autour duquel le courant de Cromwell a été observé.

M. SaINT-Guily répond que Knauss a fait des mesures sur presque tous les méridiens et notamment tout autour de l'équateur, depuis les Galapagos jusqu'au-dessus des îles Touamotou.

M. le Président remarque que, lors de sa visite à la Scripps Institution, il a été frappé par le fait que celle-ci étudiait à la fois l'océanographie et la météorologie à l'échelle synoptique. On a beaucoup étudié ces dernières années les «jet-streams». Y a-t-il une analogie entre les courants marins décrits par M. SAINT-GurLY et les «jet-streams » existant dans l'atmosphère?

M. Saint-Guny indique que la différence que l'on trouve entre la météorologie et l'océanographie vient du fait que les océans sont limités par des frontières méridiennes, constituées par les continents. M. Lacombe ajoute que l'analogie avec les «jet-streams» atmosphériques est cependant remarquable. Si, comme vient de le dire M. Saint-Guily, la présence de frontières méridiennes introduit une différence notable avec le domaine atmosphérique, l'océan Antarctique ne présente pas la limitation en question. On a avancé l'idée que la zone du courant Est fort situé à l'emplacement de la convergence antarctique pouvait être liée à un jet-stream océanique.

Sur la demande de M. le Président, M. SAINT-Guilx précise que les vitesses de $1,25 \mathrm{~m}$ qu'il a indiquées sont des vitesses movennes pendant la duree d'observation. On n'a pas encore étudié les variations mais cela semble assez stable. Il y a un effet stabilisateur à l'équateur qui doit maintenir le courant à l'équateur de façon permanente.

Mlle Gugre demande ce qu'est une « densité spectrale » et si elle est exprimée en $\mathrm{cm}^{2}$.

M. Saint-Guily répond qu'il ne s'agit pas de densité d'analyse spectrographique, mais simplement du carré de l'amplitude des oscillations observées.

M. LACOMBe pense, au sujet de la liaison hydrologiemétéorologie dont parlait $M$. le Président à l'occasion de l'exposé de M. Serra, qu'il serait heureux qu'un troisième terme soit introduit dans le cycle de l'eau, celui d' « océanographie »; car si l'eau retourne aux océans, elle en vient et le processus d'évaporation qui transfère l'eau à l'atmosphère a des effets thermiques considérables qui jouent un rôle fondamental dans la stratification thermique des couches superficielles de l'océan, en particulier dans la genèse des «thermoclines» évoquées par M. SAINT-Guily.

M. le Président remercie M. Lacombe pour ses intéres. santes interventions.

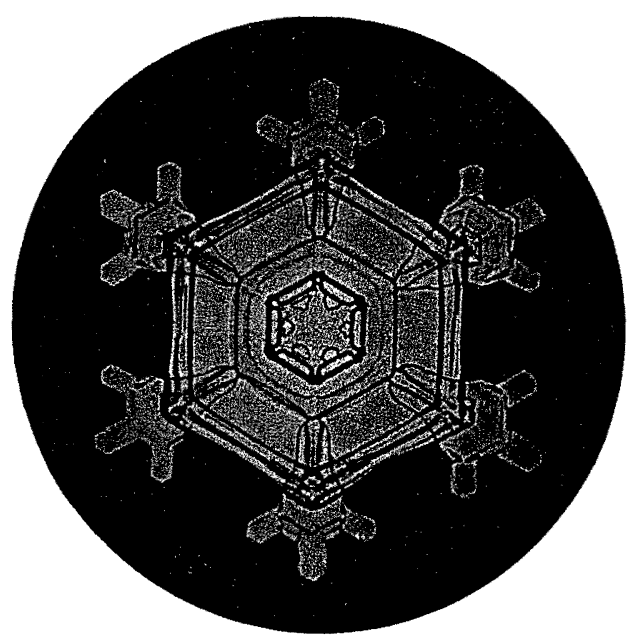

